\title{
Suppression of Radio Frequency Interferences Using the Adaptive FIR Filter Based on the Linear Prediction.
}

\author{
Dariusz Głas \\ University of Łódź \\ Department of Physics and Applied Informatics, \\ Faculty of High-Energy Astrophysics, \\ 90-236 Łódź, Pomorska 149, Poland \\ Email: dariusz.glas@uni.lodz.pl
}

\begin{abstract}
The Linear Predictor (LP) is a finite impulse response adaptive filter using linear prediction to suppress radio frequency interferences (RFI) in the Auger Engineering Radio Array (AERA). AERA focus on the electromagnetic part of the Extensive Air Showers. The electromagnetic part of the shower produces radio signals in geomagnetic radiation and charge excess processes. Due to the reflection of the atmosphere AERA radio stations can observe these signals in the frequency band 30 $80 \mathrm{MHz}$. This frequency range is contaminated by narrow-band and other human-made RFI. To suppress these contaminations AERA uses two kind of filters: the Median filter and the infinite impulse response - notch filter, however both of them have disadvantages. $L P$ is a new approach in real-time signal filtering. Laboratory and pampas tests show fast adaptation, acceptable power consumption and very good efficiency of the LP filter.
\end{abstract}

\section{INTRODUCTION}

$\mathbf{T}$ $\mathrm{HE}$ radio emission detection of extensive air showers is widely used in many experiments all over the world [1] [2]. The experiment, which focus on this task within the Pierre Auger Observatory (PAO) [3] is the Auger Engineering Radio Array (AERA) [4].

When ultra-high energy cosmic ray (UHECR) reaches the Earth's atmosphere it produces avalanches of secondary particles, called air showers. The study of the air shower allows understanding the mass of primary particle, its energy and incoming direction. These data help to understand emission mechanisms and help to find sources of the UHECR.

The Earth's magnetic field can deflect the trajectory of electrons and positrons generated in the air shower. This process generates a synchrotron radiation, which can be detected by radio stations [5] [6] [7]. Charged particles are concentrated in a thin disk in front of the shower. The coherent emission from this disk is up to $100 \mathrm{MHz}$. The frequency band from 30 to $80 \mathrm{MHz}$ was chosen due to the reflection of the atmosphere. The noise in this frequency range consists

This work was supported by the National Science Centre (Poland) under NCN Grant No. UMO-2013/08/M/ST9/00322

Dariusz Głas is the PhD student with the University of Łódź, Department of Physics and Applied Informatics, Faculty of High-Energy Astrophysics, 90-236 Łódź, Poland of galactic noise, human made continuous contamiantions and machine generated transients.

Radio detectors, installed in AERA radio stations have high angular resolution and their duty cycle is nearly $100 \%$. Moreover, they are sensitive to the evolution of longitudal airshowers and the total cost of their instalation and work is relatively low. Data taken by radio stations are next digitized by 12 bit $200 \mathrm{MHz}$ analog-to-digital converter and processed in real time by FPGA chip. The triggering depends on a signal shape, so it requires good-quality data. The filtering of the signal is crucial.

\section{MathematicAl BACKGROUND}

The Linear Predictor (LP) is an adaptive finite impulse response (FIR) filter based on the linear prediction. If the periodic signal is hidden in data, we can use several data samples (stages of the filter) to predict how it would look like in the future [8]. Next, we can subtract the predicted periodic signal from the original one, to get the clean data. The graphical presentation of this process is shown on fig. 1 .

If the $s(i)$ represents the continuous stream of data samples, the $a_{n}$ are the filter coefficients and $e(i)$ is cleaned signal, we can describe the LP filter as:

$$
e(i)=s(i)-\sum_{n=1}^{p} a_{n} s(i-D-n),
$$

where $p$ is the number of used data samples and simultaneously number of the coefficients of the LP filter, $D$ is the delay between data we used for prediction and data we want to predict. This delay is necessary to minimize the distortion of the transient signals. It would be discussed later. The predicted signal $\hat{s}(i)$ can be described as:

$$
\hat{s}(i)=\sum_{n=1}^{p} a_{n} s(i-D-n) .
$$

We can find the optimal solution of the coefficients by 


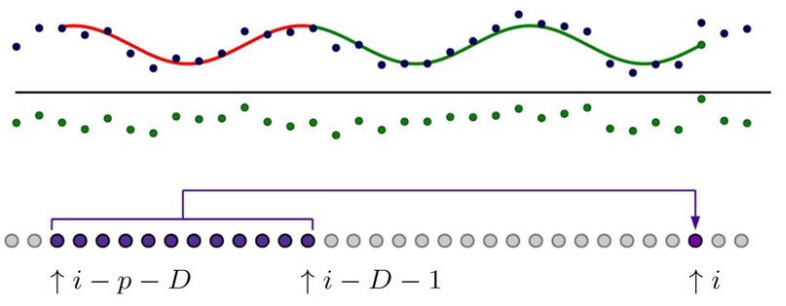

Fig. 1. Graphical illustration of algorithm of the LP filter. Several stages of data are used to predict how the periodic signal hidden in data will look like in the future (above the line). Filtered signal (below the line) is received after subtraction predicted signal form the original one.

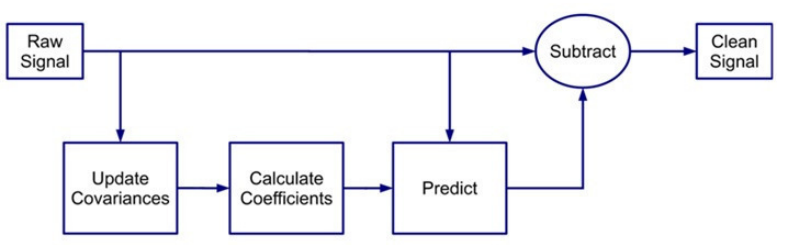

Fig. 2. Schematics showing the LP filter data flow in FPGA. After updating covariance matrix, coefficients can be found by solving a system of linear equations. Prediction step is just summing the products of the coefficients and delayed data. This predicted signal is used for subtraction from original data samples, to get filtered signal.

assuming the Gaussianity and calculating the estimated mean square error:

$$
E=\frac{1}{N} \sum_{i=0}^{N-1} e^{2}(i)=\frac{1}{N} \sum_{i=0}^{N-1}(s(i)-\hat{s}(i))^{2},
$$

where $N$ is number of data samples used for calculation of the covariance matrix. In our tests we used $N=1024$. The next step in finding the best values of coefficients is the minimization of the mean square error, by derivating it with respect to every coefficient and comparing the result to zero:

$$
\frac{\partial}{\partial a_{i}} E=0
$$

As a result we get the system of $p$ equations:

$$
\sum_{i=0}^{N-1} s(i-D-n) s(i)=\sum_{i=0}^{N-1} \sum_{m=1}^{p} a_{m} s(i-n) s(i-m) .
$$

We can rewrite this equation in vectorial form:

$$
\overrightarrow{r^{*}}=\boldsymbol{R} \vec{a}
$$

where $\vec{a}$ is vector of the coefficients and $\overrightarrow{r^{*}}$ is defined as follows:

$$
r^{*}(n)=\sum_{i=0}^{N-1} s(i-D-n) s(i) .
$$

$\mathbf{R}$ is the covariance matrix described as:

$$
R(m, n) \equiv \sum_{i=0}^{N-1} s(i-n) s(i-m) .
$$

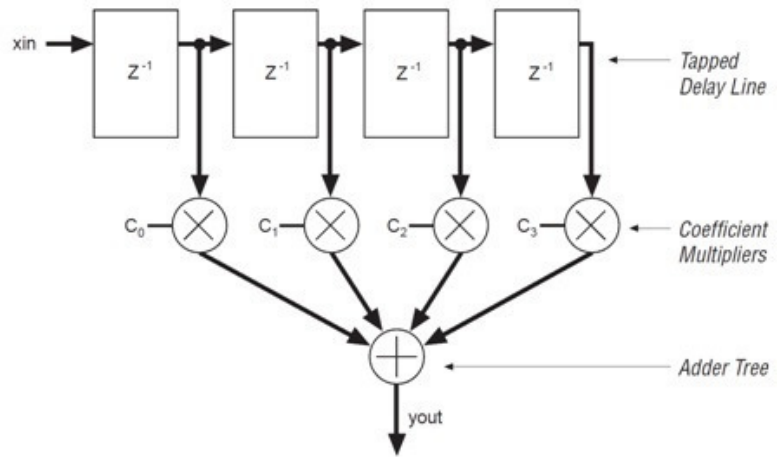

Fig. 3. Schematics of prediction step. Delayed data are multiplied by proper coefficients. Products are summed by several steps of accumulation.

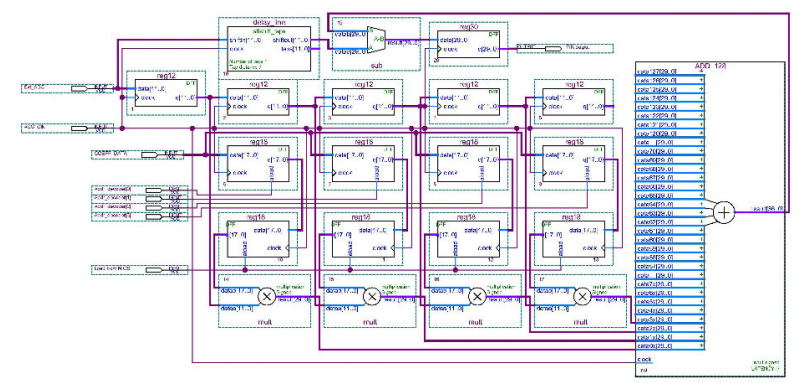

Fig. 4. Fragment of fast logic block connections required for the prediction step.

This matrix is symmetric and diagonal-constant (Toeplitz matrix). The system of linear equations consisting on the Toeplitz matrix can be solved using Levinson procedure instead of Gauss-Jordan elimination. The Levinson procedure algorithm runs in $o\left(n^{2}\right)$ time, which is significant improvement in comparison to Gaussian algorithm, which runs in $o\left(n^{3}\right)$.

\section{FPGA IMPLEMENTATION}

Fig. 2 shows the FPGA data flow of the LP filter. The 1024 ADC samples are used to build the covariance matrix. This step can be done either in the virtual processor NIOS ${ }^{\circledR}$ or in fast logic blocks. The creation of the matrix and the $\left(\overrightarrow{r^{*}}\right)$ vector in fast logic requires the delay line to get $s(i-D-n)$, the multiplier to get the partial terms and the accumulator. The covariance matrix can be created in fast logic blocks in time of $5 \mu \mathrm{s}$, when in the NIOS $^{\circledR}$ it can be done in several tens of ms. The main reason of this is relatively slow data transfer to the $\operatorname{NIOS}^{\circledR}$. When virtual processor NIOS $^{\circledR}$ receives the data, it sends the signal, that it is ready to receive the next data and the address of this data in DPRAM. This process is very time consuming. Moreover, the NIOS ${ }^{\circledR}$ processor can work with maximal frequency only $100 \mathrm{MHz}(80 \mathrm{MHz}$ for tests). The fast logic works with $200 \mathrm{MHz}$ clock and can be used in situations, when time is an important factor.

The calculation of the LP coefficients is the most time consuming step in the refreshing of the LP filter. It cannot be done in the fast logic due to a complexity of the task, 

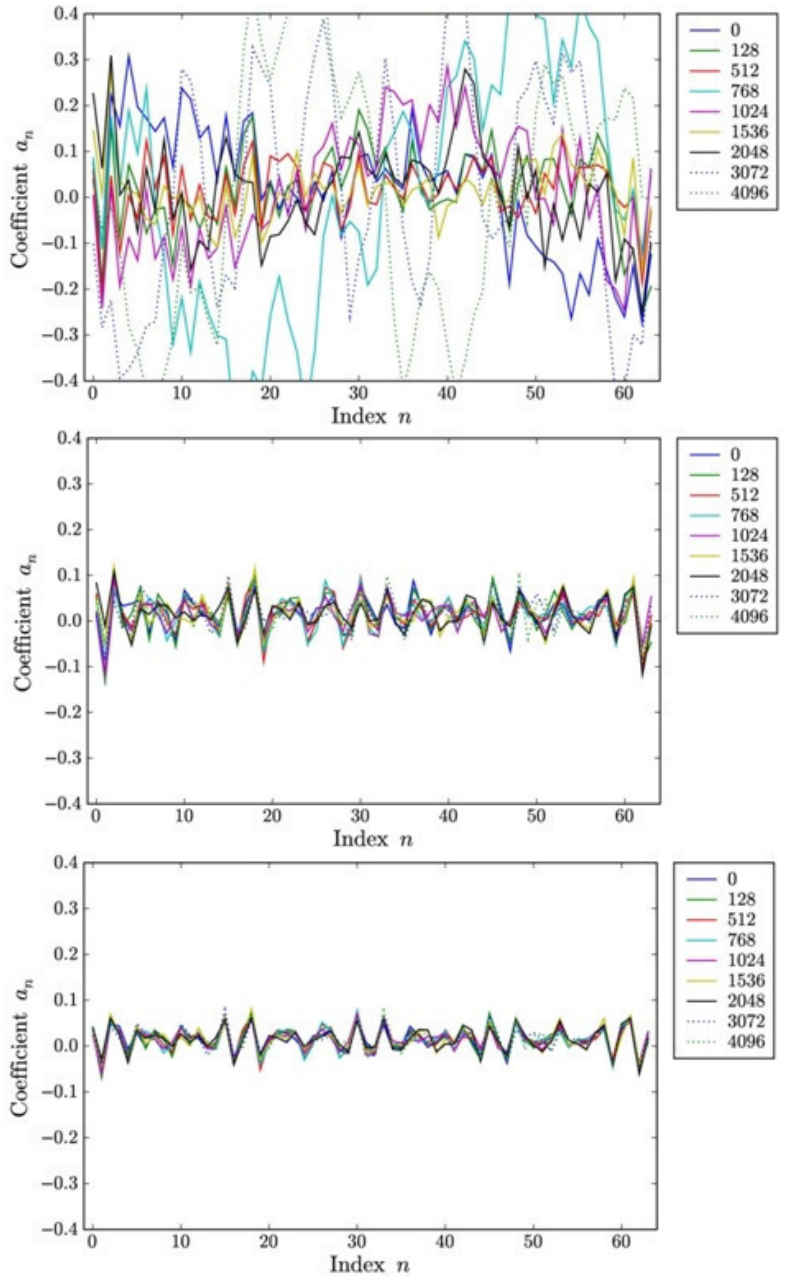

Fig. 5. Stability of the calculated coefficients. Upper plot shows the situation when the determinant of the covariance matrix is close to zero. After introducing the fudge factor $f$ coefficients are stabilized slightly (middle graph $f=0.1$ ). For $f=1$ (bottom plot) the stabilization is the best.

but it can be done in the virtual processor NIOS ${ }^{\circledR}$. This step consists in solving the vectorial equation (eq. 6) and highly depends on the number of coefficients and the method used for solving. The Levinson method needs $191 \mathrm{~ms}$ for solving the $64 \times 64$ covariance matrix, when the Gauss elimination for the same task requires almost 2 seconds. For solving the $128 \times 128$ covariance matrix Levinson procedure needs $760 \mathrm{~ms}$ and Gauss elimination almost 15 seconds.

Results of both methods are the same. After the calculation, coefficients are transferred from the $\mathrm{NIOS}^{\circledR}$ to fast logic temporary registers one by one, which requires at least $p$ clock cycles, where $p$ is the number of the coefficients. When all coefficients are transferred to temporary registers, they are reloaded in one clock cycle to destination registers. This step is required to avoid the situation, when some of coefficients are "old" and some are "new".

The prediction step consists in summing multiplications of coefficients and delayed data (eq. 2). This step is done in

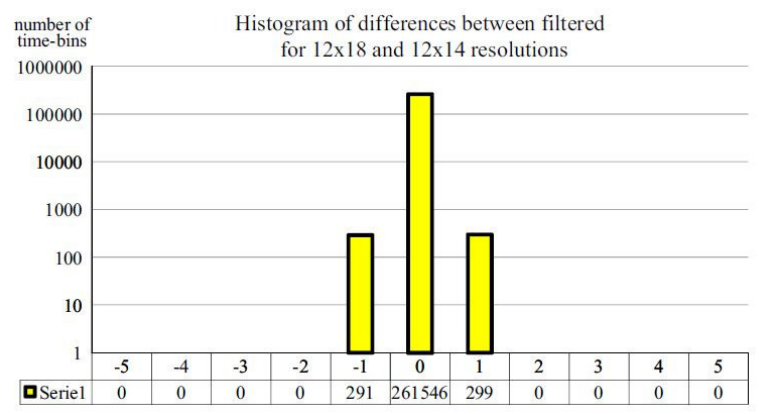

Fig. 6. Histogram of differences between $12 \times 14$ and $12 \times 18$ resolutions. Differences shows only on the LSB and are on the level of $0.01 \%$.

FPGA fast logic. Data are delayed to get $s(i-D-n)$ and next they are multiplied by an appropriate coefficient. This product is next added in several steps to get the predicted, periodical signal, hidden in the original data. Fig. 3 shows the schema of this process and fig. 4 shows fast logic blocks connections required for calculations. The prediction step can work continuously, because it does not require the gap to update the coefficients. After the prediction we just have to subtract the predicted, periodic signal from the original one to get the clean data.

\section{OPTIMIZATION OF THE LP FILTER}

The stability of the coefficients in time is shown on the fig. 5. The instability of the coefficients seen in the first plot is caused by the specific situation, when determinant of the covariance matrix is close to zero. To avoid this situations we introduce the fudge factor $(f)$ and change the covariance matrix:

$$
\begin{gathered}
\tilde{R}_{n n}=(1+f) \cdot R_{n n} \\
\tilde{R}_{m n}=R_{m n} \text { for } m \neq n
\end{gathered}
$$

Middle and bottom graphs show the stabilization of the coefficients for $f=0.1$ and $f=1$ respectively. The stabilization is the best for The next specific parameter is the width of coefficients data. The algorithm of the LP filter requires multiplications of coefficients and data from the ADC. The dedicated multiplier requires the different number of DSP blocks, depending on data width. Single DSP block can be used if ADC data width and coefficients width are maximally 9 bits, however data received from the ADC is 12 bits. This implicates the multiplier must use at least 2 DSP blocks. Using 2 DSP blocks allow to multiply 12 bit data with maximally 18 bit coefficient. The 18 bit accuracy of coefficients is not necessary, so it can be reduced to 14 bits without special loses in the efficiency. Differences between those two cases for the filtered signal are only on the LSB and appear to be on the level of $0.01 \%$ (fig. 6). Moreover, the reduction from 18 to 14 bits reduces by $\sim 30 \mathrm{~mW}$ the power consumption. The further reduction of the coefficients width (to 12 bits) increases differences to the level of $1 \%$. The reduction of the power consumption is only 

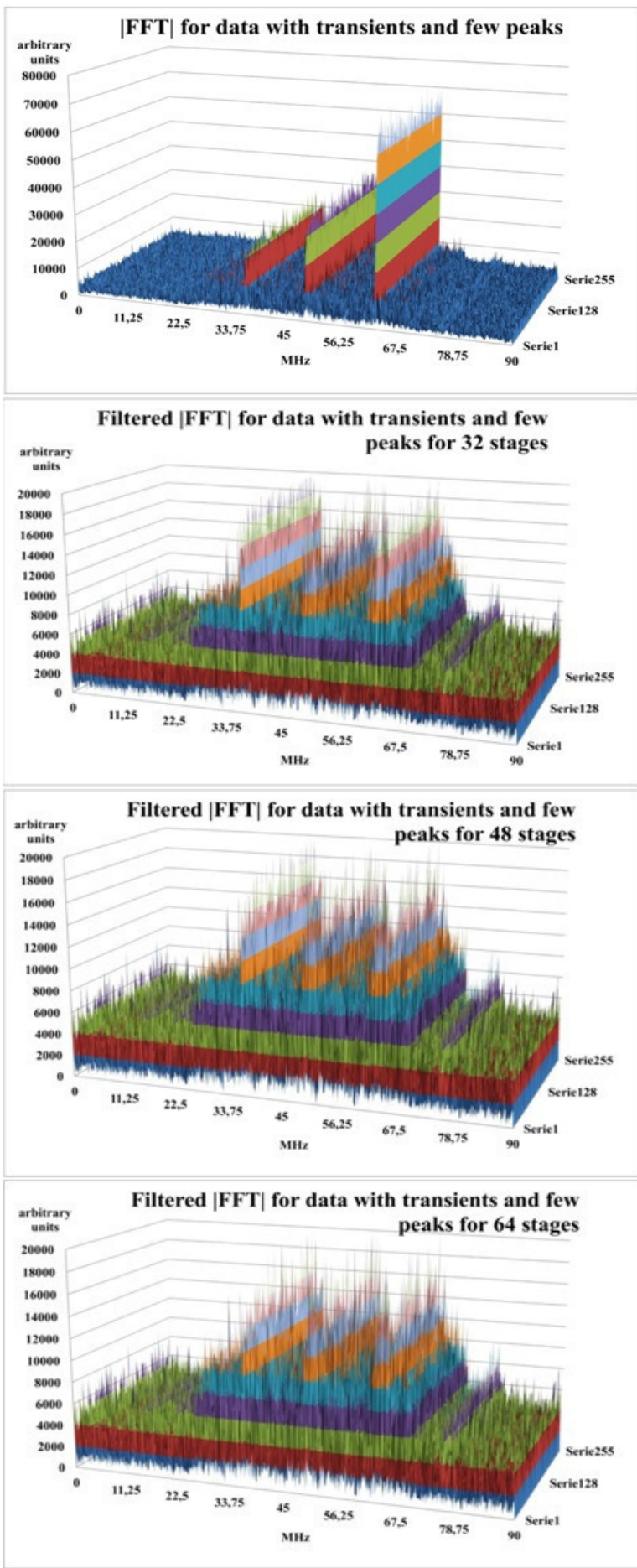

Fig. 7. Original and filtered data with transients and few peaks. LP filter variants with 32, 48 and 64 stages give almost the same result. Slightly contaminated data can be filtered using LP filter with 32 stages

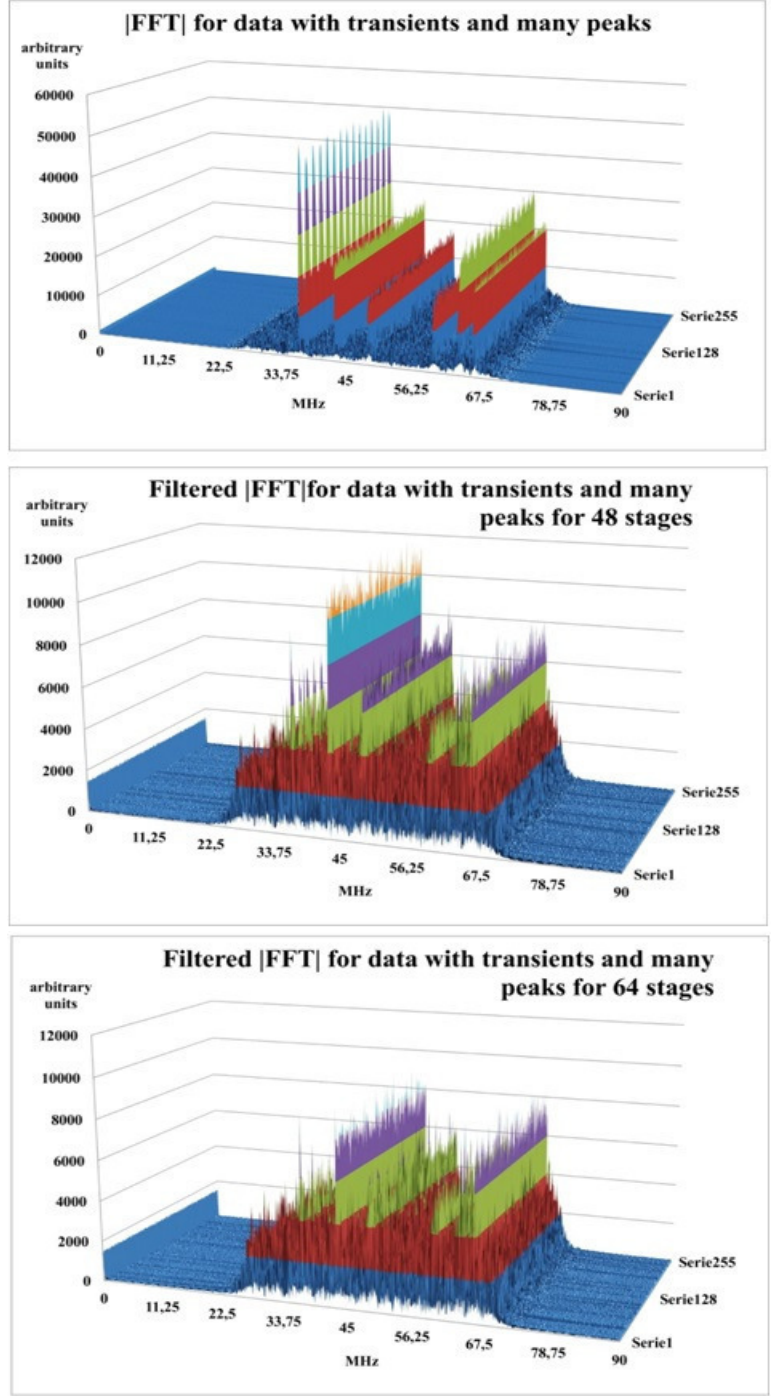

Fig. 8. Original and filtered data with transients and many peaks. In this case the suppression factor is much better in 64-stages variant of the LP filter, however variant with 48 stages also significantly suppresses the contamination. It is recommended to use 64 stages for strongly contaminated data.

$\sim 10 \mathrm{~mW}$, which is negligible. It was decided that width of the coefficients would be 14 bits.

The length of the filter is an important parameter, which has significant influence on the efficiency of the LP filter. The length of the filter is the number of data used for the prediction and it is the same as the number of coefficients of the filter.

Various lengths of the filter: 64, 48 and 32 have been tested. The filter with length 128 was decided not to be tested, because of the long time of the refreshment (almost $0.8 \mathrm{~s}$ ). Results of tests are shown on fig. 7 and fig. 8. Fig. 7 shows the data contaminated only by few narrow RFI. The results of the filtering for this case are almost the same for 64,48 and even for 32 stages of the filter. However, when data are contaminated by many RFI (fig. 8) the filtering using 64 coefficients is more efficient than using 48 stages of the 

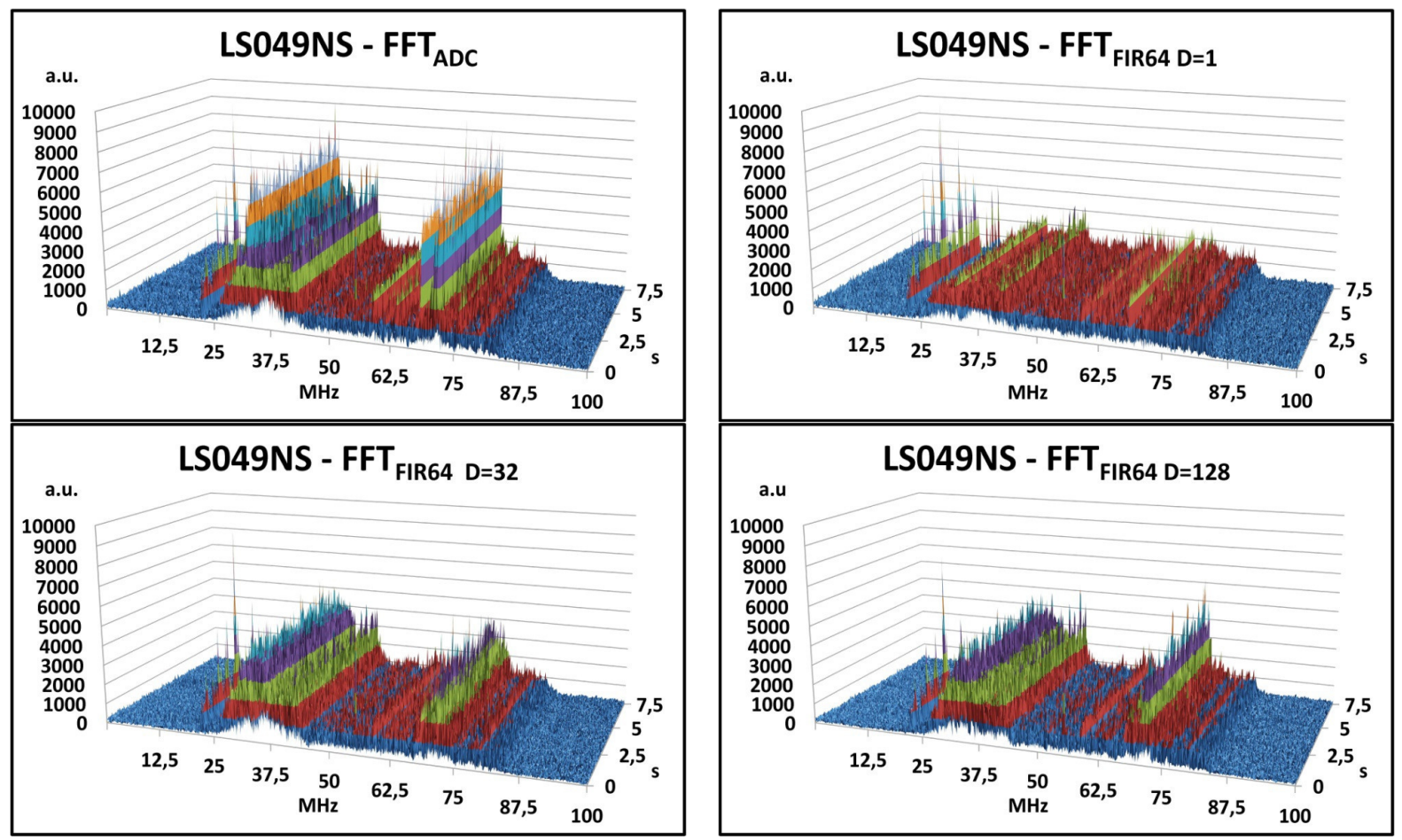

Fig. 9. $|\mathrm{FFT}|$ of original and filtered data from station LS049, polarization NS for several variants of delay line $D$. Filtering is the best, when the delay line $D=1$. In rest of cases filtering is on the same, acceptable level.
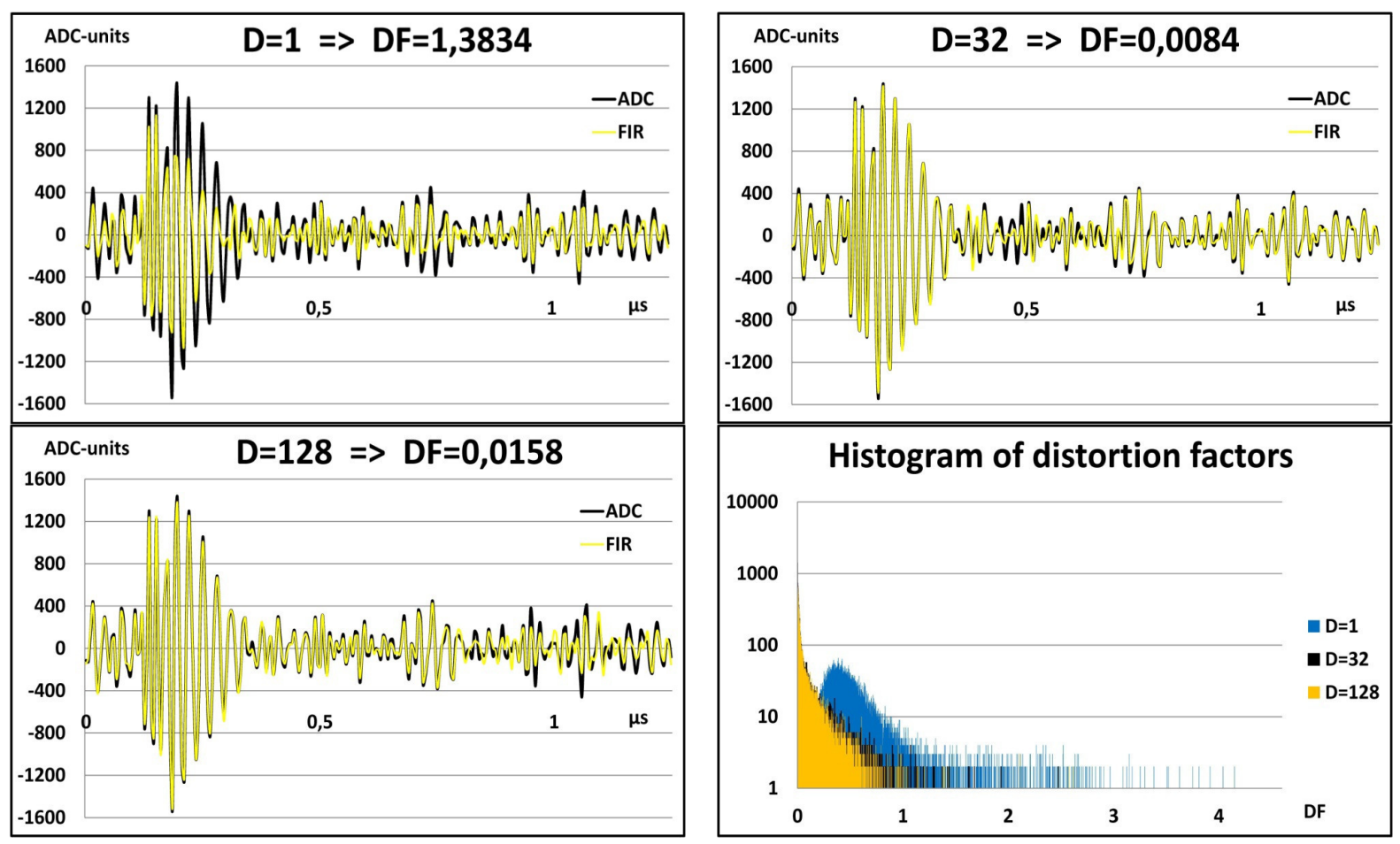

Fig. 10. Original and filtered data in time domain. Although suppression of the variant of the LP filter with delay-line $D=1$ is the best, it distorts signal too much and has to be rejected. Both variants, with 32 and 128 length of the delay-line move the distortion made by signal appropriately 32 or 128 data samples away from the signal. In these cases signal remains almost unaffected. 

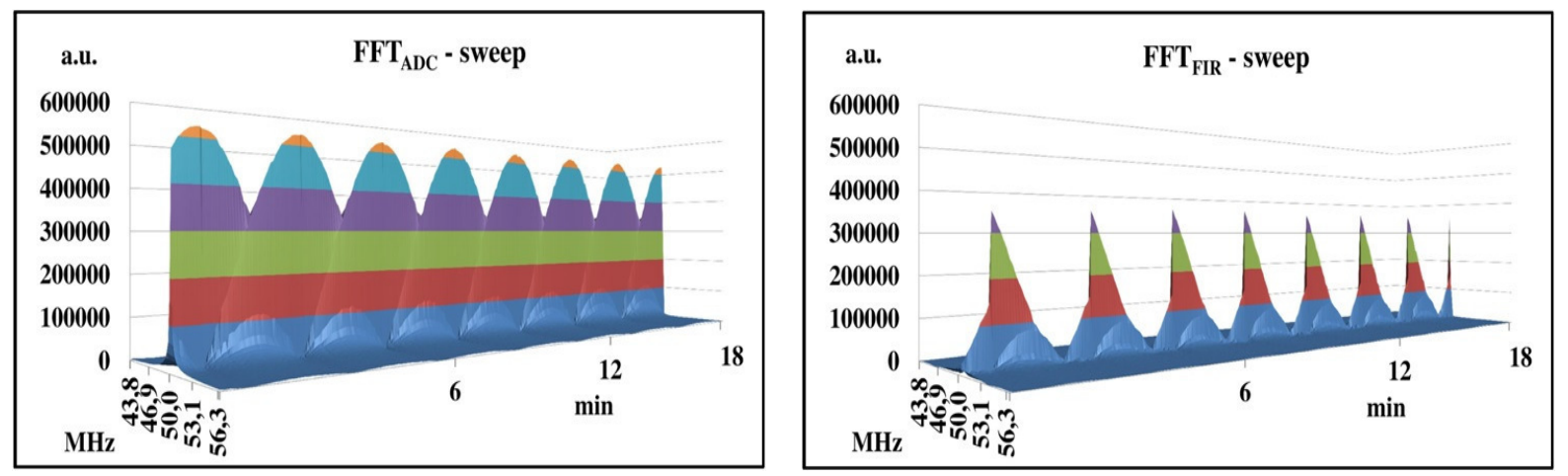

Fig. 11. IFFTl of original and filtered mono-carrier with 2 minutes drift in frequency from $50.0 \mathrm{MHz}$ to $50.2 \mathrm{MHz}$. When frequency of the contamination is the same as in time of calculation coefficients for the LP filter, suppression level is very high. This result is repeated every 2 minutes.
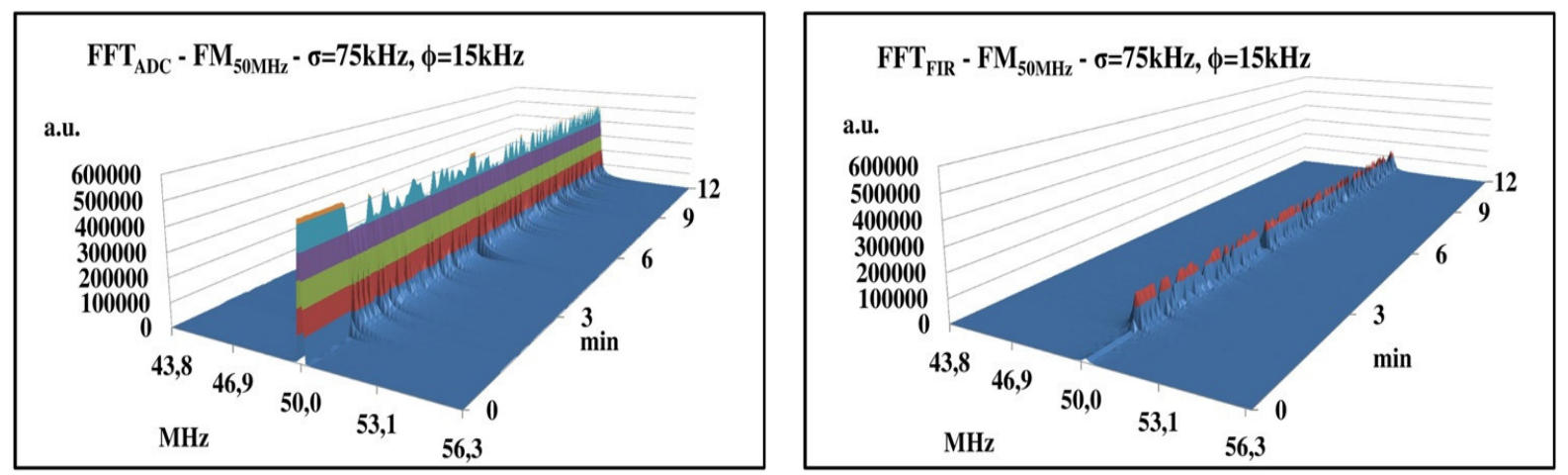

Fig. 12. IFFT| of original and filtered mono-carrier with restrictive FM modulation. The efficiency of the filtering is still very high.

filter. Shorter filters require less FPGA resources, use less power and can be calculated much faster ( $\sim 2$ times faster for 48 stages and $\sim 4$ times faster for 32 stages of the filter). Simulations show, the LP filter can be used with 32 stages in situations, where the contamination is not sophisticated. For very contaminated data we can use the LP filters with 48 or 64 stages.

The delay line $D$ between the data we used for prediction and data we want to predict was introduced to avoid the distortion of the signal. We can define the distortion factor $(D F)$ to calculate how the filter affects data and the registered signal:

$$
D F=\sum_{k=-16}^{16}\left(\frac{\left(x_{F I R}\right)_{k}-\left(x_{A D C}\right)_{k}}{\left(x_{A D C}\right)_{k}}\right)^{2}
$$

Fig. 9 shows filtered data for three variants of parameter $D$. For all values of the $D$ factor the LP filter shows good efficiency, but filtering is the best when data we want to predict is located just after data we used for prediction $(D=1)$. However, this case introduce significant distortion of the signal (fig. 10 upper left plot) and has to be rejected. Histogram of distortion factors (fig. 10 bottom right plot) shows explicitly, that delay-line $D=128$ distorts signal minimally. $D=128$ moves the distortion made by signal 128 data samples away from the signal region. To keep this safety margin, it was decided to use delay-line $D=128$ for all tests.

\section{LABORATORY TESTS}

All laboratory tests were made using Altera DK-DEV5CEA7N development kit with Cyclone V FPGA. The generated signals were transferred from generators to Texas Instr. ADS4249EVM Evaluation Module with 2-channel 14-bits 250 MSps ADC. Connection between both modules was provided using the LVDS data transmission by Altera HSMC-ADCBRIDGE (fig. 22).

The long-term stability of the filter was tested by using a single mono-carrier, which frequency drifted from $50.0 \mathrm{MHz}$ to $50.2 \mathrm{MHz}$ in 2 minutes. Coefficients of the LP filter had been calculated and had been loaded to the registers before this test. These coefficients were not changed during this test. Fig. 11 shows results of the test in time of several minutes. We can observe very good suppression when the frequency goes back to the frequency, which was used for calculation of LP filter coefficients. If data are contaminated by RFI which frequency slowly changes in time, coefficients of the LP filter do not have to be refreshed frequently.

Next test consists on checking the Hi-Fi FM configuration. The signal was contaminated by $50 \mathrm{MHz}$ carrier with $75 \mathrm{kHz}$ deviation, with acoustic modulation $15 \mathrm{kHz}$. This conditions 

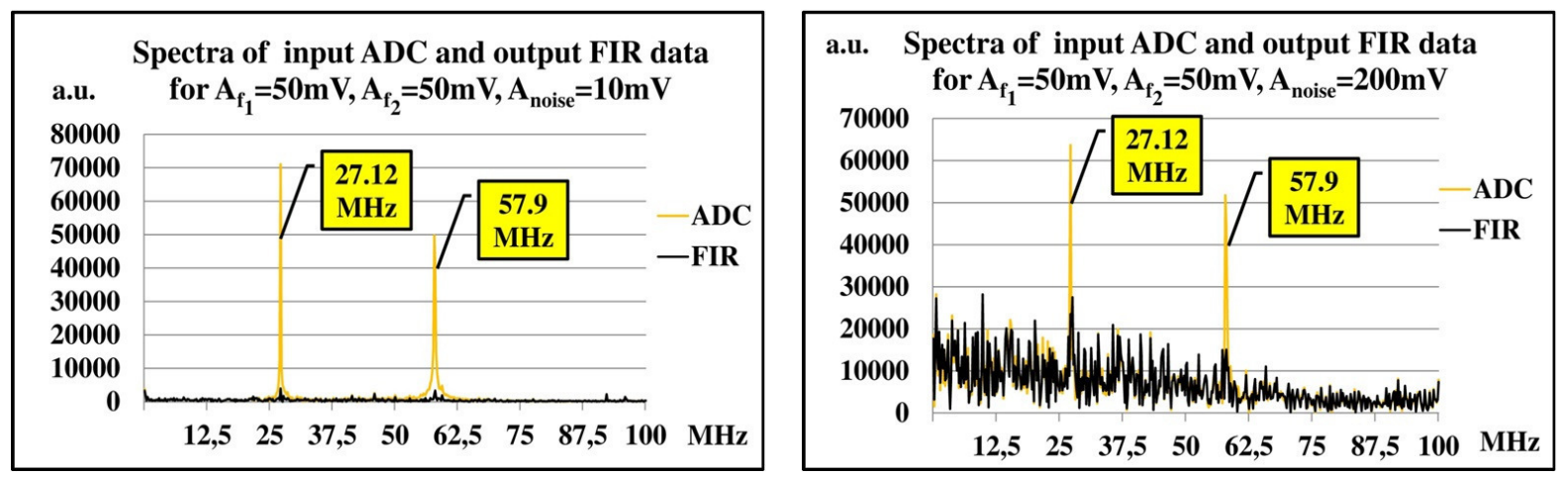

Fig. 13. IFFT| of original (ADC) and filtered (FIR) data of two mono-carriers with 2 variants of noise level. Suppression of carriers is on very good level, even if the amplitude of the noise is greater than the amplitude of carriers.

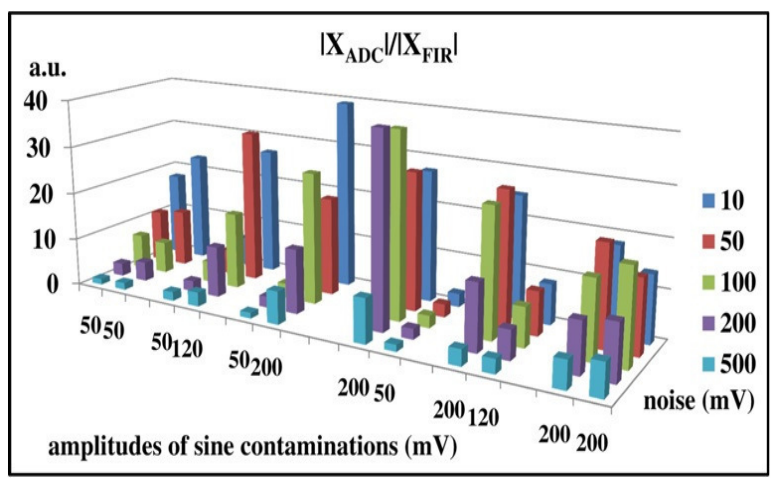

Fig. 14. Suppression levels of two sine contamination in dependancy of noise level and several variants of sine amplitudes. Noise level can greatly change the suppression factor. Contamination with higher amplitude is much more suppressed than the second one. This effect is not dependant on noise level.

are very restrictive and they should not appear in real conditions in detectors on pampas. Frequency band for Hi-Fi FM transmissions is $88-108 \mathrm{MHz}$ and it is cut-off by already installed analog filters. The suppression of these restrictive contamination is very good (fig. 12).

The LP filter was also tested to check the suppression efficiency. The generated signal was two carriers (27.12 and 57.9 MHz) with noise. Suppression was almost total, when signals were much stronger than noise, but there was also a significant suppression when signals and noise were on the same level (fig. 13).

Several variants of the carriers and noise amplitudes had been tested. Fig. 14 shows the dependency of the suppression level on amplitudes of contamination. Higher noise level reduces efficiency of filtering. We can also observe, that if the amplitude of one carrier is much higher than the amplitude of the second carrier, the suppression level of the second carrier is much lower than the suppression level of the first carrier.

\section{COMPARISON TO THE CURRENTLY USED FILTERS}

\section{A. Median filter}

AERA uses two kinds of filters to improve the signal to noise ratio. The first kind is the Median filter. It is based on the Fast Fourier Transform (FFT) technique. Radio signals are transferred from the time domain to the frequency domain by FFT. In the next step, the Median filter suppresses narrow peaks, which correspond to stationary mono-frequent contaminations. This filtering does not affect the radio pulses from cosmic showers. After this step, the cleaned signal is transformed to the time domain again by using the inversed FFT. The whole process is shown on fig. 15. The Median filter has also several disadvantages. Theoretically, the chain of the FFT and inversed FFT should give same data. However, due to finite representation of data in the FPGA, this chain of transformations introduces approximation errors. There are two possible architectures of the FFT: streaming and variable streaming. The streaming architecture uses less resources and is faster than the variable streaming, but the approximation error is much bigger. In the variable streaming architecture the error is reduced to the last significant bit. Another problem is aliasing. When the pulses are located near the converted data blocks, the reconstructed signal may change. Aliasing elimination can be done using overlapping neighboring data blocks, which require over-clocking of the signal processing. This approach is much more energy and resource consuming. The Median filter also distorts the signal in the region near the peak, which is responsible for the trigger. Fig. 16 shows the distortion factors of the Median filter and the LP filter for comparison. We can see, that the LP filter distorts the signal less than the Median filter. Another issue is the power consumption, which can be on the level of $1 \mathrm{~W}$ per channel. The power consumption is an important factor for experiment powered by solar panels.

\section{B. Notch filter}

Notch filter is infinite impulse response (IIR) filter, which is currently used by AERA. Its power consumption is much lower than the power consumption of the Median filter, which is important for the experiment powered by solar panels. Notch filter is not adaptive and can reject only four arbitrary chosen frequencies from the signal. After programming in the FPGA, these frequencies cannot be changed dynamically, because of potential instability of the filter. These selected frequencies 


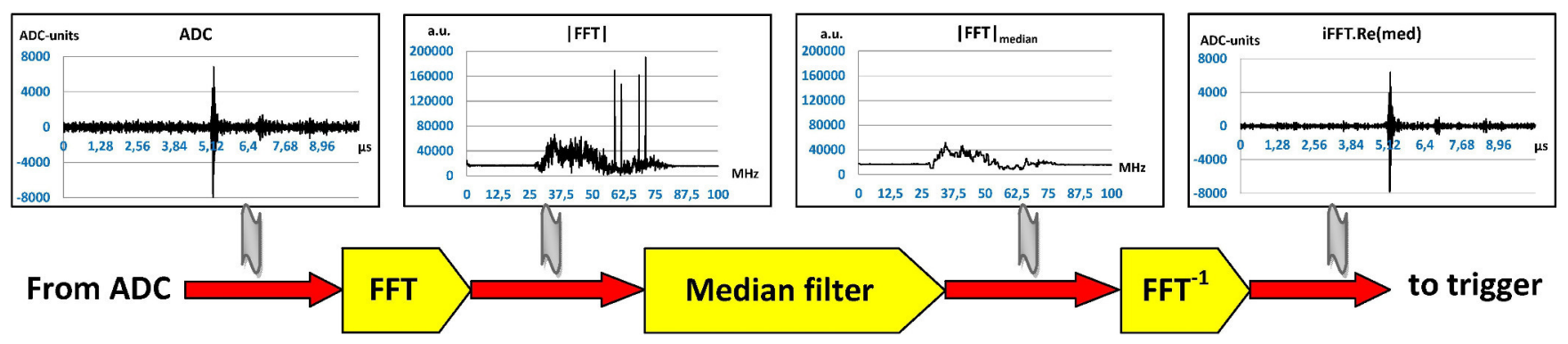

Fig. 15. Diagram showing the data flow in Median filter. Input signals are transfered to frequency domain by FFT and after the filtering by Median filter, cleaned signals are transfered to time domain again using inversed FFT.

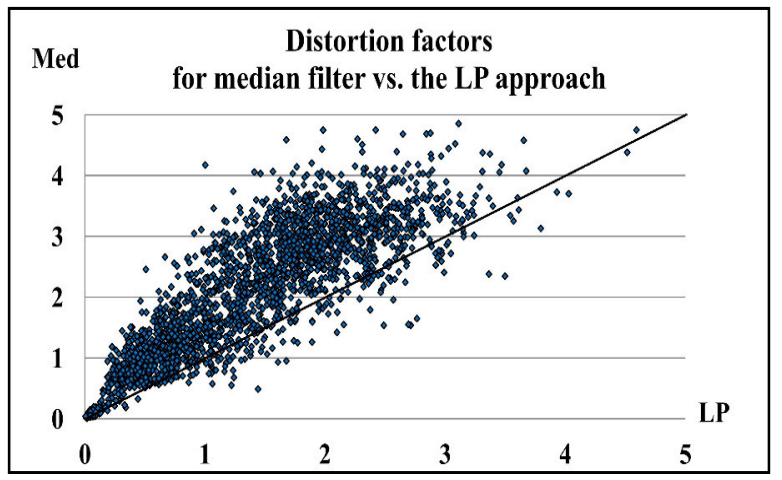

Fig. 16. Comparison of distortion factors for Median and LP filters. In most cases the LP filter distorts the signal less than the Median one.

were found by averaging data from entire array. Fig. 17 shows the comparison of the Notch and LP filters. The efficiency of the Notch filter is very high only near rejected frequencies. Suppression outside the rejected band is much lower. The current consumption of the IIR and the several variants of the LP filter is shown on the fig. 18. The variant with 32 stages has the power consumption compared to the IIR filter. Despites of their limitations, Median and Notch filters were implemented and have successfully worked in the AERA radio stations for several years.

\section{HPS IMPROVEMENTS}

The laboratory tests shows high efficiency of the LP filter, however noise on pampas is much more sophisticated. Besides of narrow-band mono-carriers tests show additional, nonstationary RFI. This type of contamination is weakly affected by the LP filter and strongly decreases filters efficiency. Currently used Notch filter also cannot suppress this kind of contamination, because it can only reject fixed frequency RFI. The main reason of weak suppression by LP filter is probably too long refreshment time of filter coefficients. In laboratory tests, coefficients were calculated by virtual processor NIOS ${ }^{\circledR}$. This processor can work with maximal frequency $100 \mathrm{MHz}$, but for tests this frequency was lowered to $80 \mathrm{MHz}$ (to have safety margin). Refreshment time of the filter coefficients in these conditions is $190 \mathrm{~ms}$. The LP filter can suppress RFI, which are longer than the time of its refreshment (fig. 19

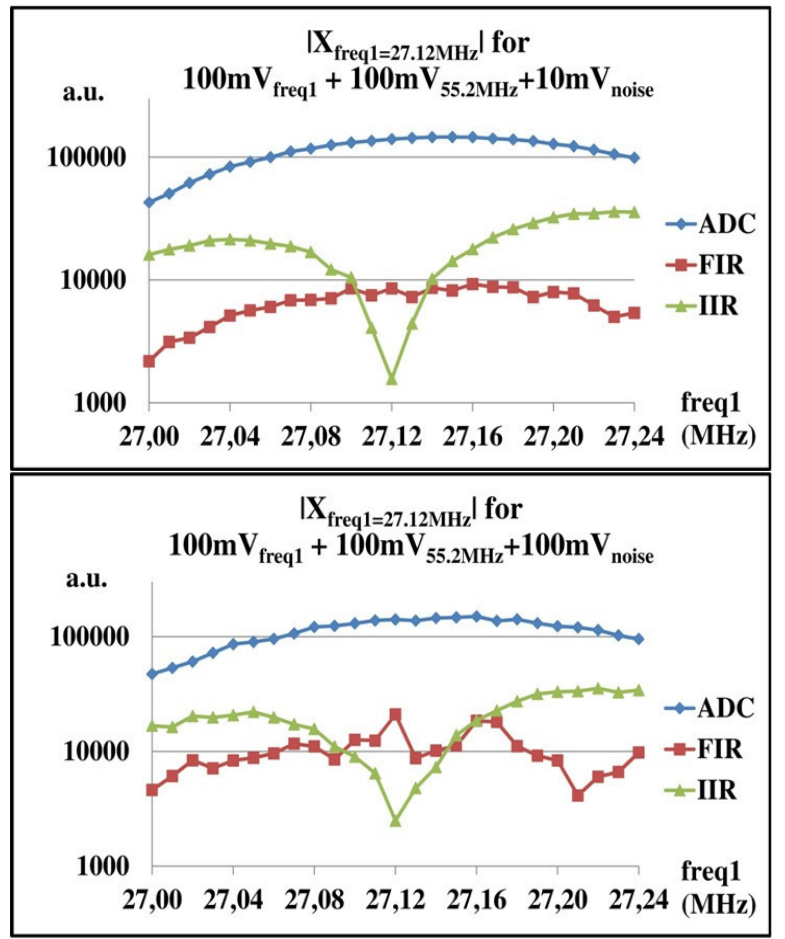

Fig. 17. Comparison of efficiency of LP FIR filter and IIR Notch filter for two variants of noise level. Suppression factor of the Notch filter is more efficient than LP filter only in narrow frequency band near the rejected frequency. Higher noise slightly decreases efficiency of the FIR filter.

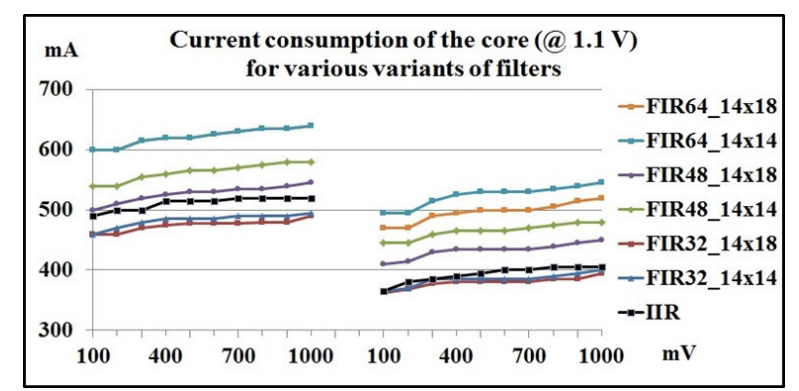

Fig. 18. The current consumption calculated for several variants of the LP filter and for currently used IIR Notch filter. Power consumption for LP filter with 32 stages is on the same level as for IIR filter. 

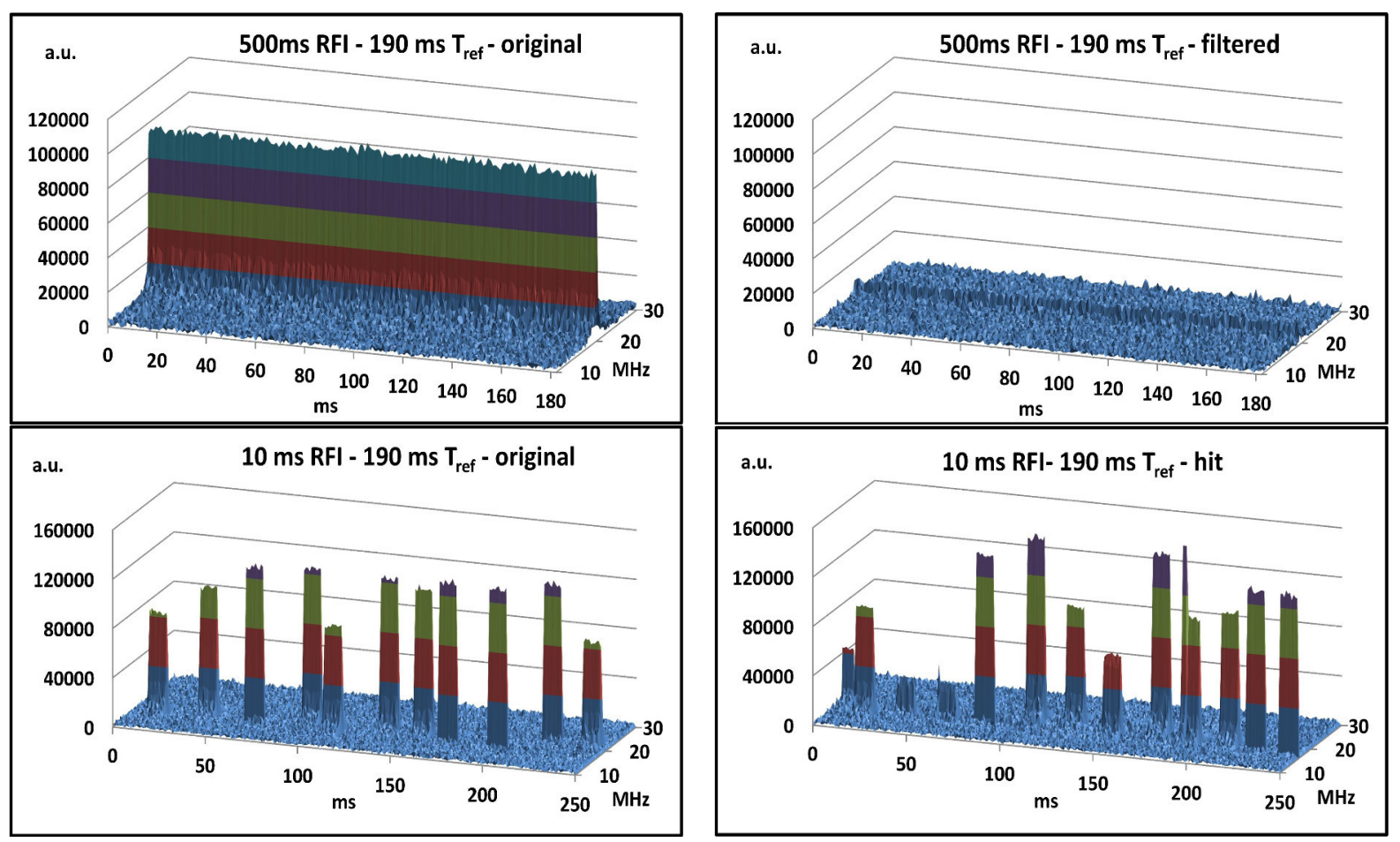

Fig. 19. Suppression achieved by LP filter with coefficients calculated in $190 \mathrm{~ms}$ by NIOS ${ }^{\circledR}$ for signals much longer (upper plots) and much shorter (bottom plots) than the refreshment time of the filter. In first case, the suppression is almost total. In second case suppression depends on similarity of data used for calculation and currently filtered data.

upper graphs). If the RFIs length is shorter than refreshment time of LP coefficients, the suppression factor depends on the similarity the signal on which the coefficients were calculated to signal, which is currently filtered (fig. 19 bottom graphs). We can define the suppression factor:

$$
S F=\frac{\sum F F T_{\text {original }}-\sum F F T_{\text {filtered }}}{\sum F F T_{\text {original }}} .
$$

To reduce the non-stationary RFI, coefficients of the LP filter have to be calculated more frequently. To achieve this we can use Cyclone V SoC HPS, which contains embedded ARM processor. This processor can work with maximal frequency $925 \mathrm{MHz}$. To have the safety margin, all laboratory tests were made using $800 \mathrm{MHz}$. Fig. 20 shows the calculation time of the coefficients for two variants of the LP filter. Refreshment time can be reduced from $190 \mathrm{~ms}$ to several milliseconds. Fig. 21 shows the simulated results of the filtering by this filter for three different lengths of RFI. The efficiency of the filter is very high for all tests. Suppression factors for case with 100 ms length RFI is $\mathrm{SF}=72 \%$ and for $50 \mathrm{~ms} \mathrm{RFI} \mathrm{SF}=59 \%$. If the coefficients were calculated during the gap between the signals, the filter does not suppress the signal. It is seen on all graphs containing filtered data. In $50 \mathrm{~ms}$ and $20 \mathrm{~ms}$ RFI one of peaks is increased. The probable reason is a short gap between two peaks, so old coefficients were calculated using the data from the previous peak. Every peak is independent, so filter may not work properly in this case. If the refreshment time of LP coefficients is slightly lower than the length of the

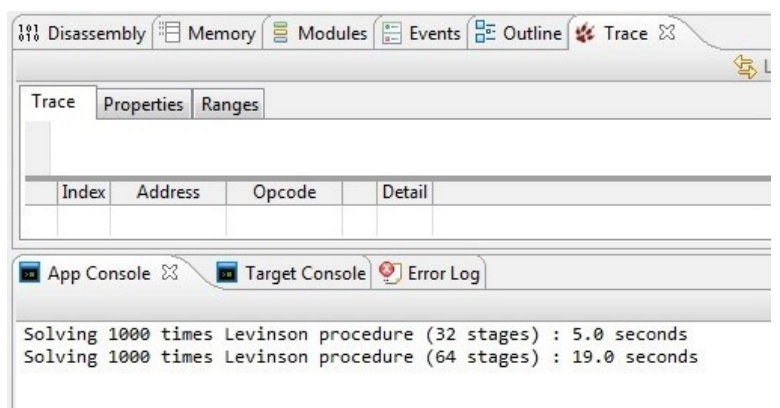

Fig. 20. Calculation time for solving by ARM processor system of 32 and 64 linear equations for two variants of LP filter using Levinson algorithm. Calculation time can be shorten from $190 \mathrm{~ms}$ to several ms.

RFI (fig. 21 bottom graphs), suppression is highly dependent on the moment of starting the calculation of new coefficients. The best scenario is when the calculation starts just after new RFI shows. New set of coefficients starts working near the middle of the peak and can significantly suppress rest of the peak. If the calculation use data just before the new RFI, next calculation would be done in the middle of the RFI occur and starts working at the end or just after the RFI. The suppression factor for this case is much lower than in longer RFI cases, but it is still on acceptable level $\mathrm{SF}=24 \%$.

The communication between the FPGA and ARM processor is much faster than between the NIOS ${ }^{\circledR}$ and FPGA. The 128 bit bridge between the ARM and FPGA allows sending up 

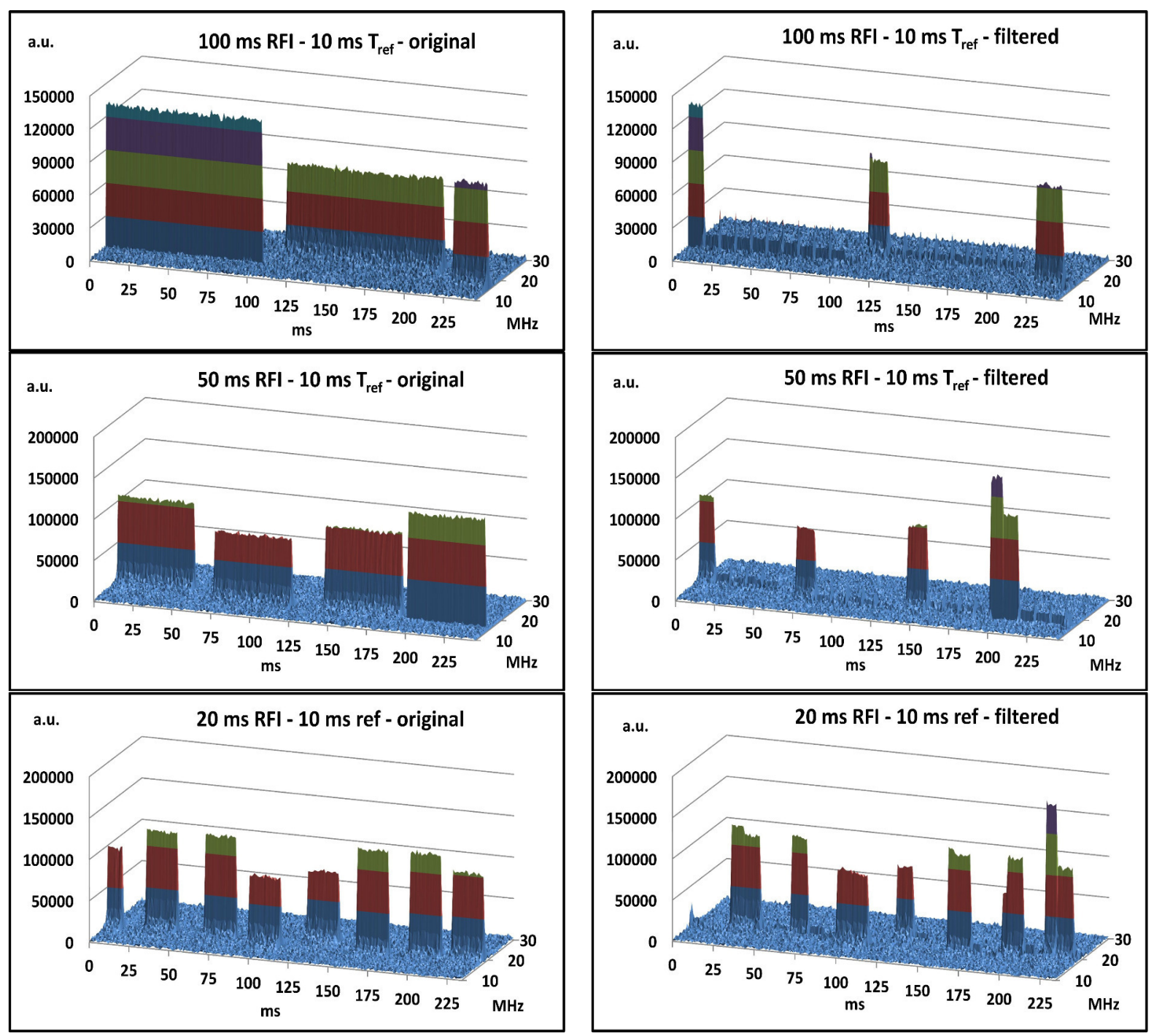

Fig. 21. Suppression achieved by LP filter with coefficients calculated in $10 \mathrm{~ms}$ by ARM processor for signals much longer (upper plots) and slightly longer (middle and bottom plots) than the refreshment time of the filter. In upper and middle graphs suppression is very high. The filter does not work only when its coefficients were calculated during the gap between peaks. In bottom graphs suppression strongly depends on the moment of coefficients calculation.

to 10 coefficients in one clock cycle. This means that 64 coefficients can be send in 7 clock cycles and 32 coefficients in 4 clock cycles. Virtual processor NIOS ${ }^{\circledR}$ allows to send only one coefficient per clock cycle.

\section{CONCLUSIONS}

The Linear Predictor is a new kind of filter, which can be used in AERA radio stations. Fast adaptation to new environment conditions, high efficiency in suppression contaminations and reasonable power consumption show it can succesfully replace currently used Notch and Median filters. Additionally, the LP filter leaves almost unaffected signal used for triggering. Using the ARM processor allows to suppress very short non-stationary RFI, which are not suppressed by currently used filters. Moreover, the LP filter is very flexible. The shorter versions of the filter can be implemented in radio stations, where RFI is relativelly weak. Longer versions can be used in radio stations, where filtering is crucial.

\section{ACKNOWLEDGMENT}

This work was supported by the National Science Centre (Poland) under NCN Grant No. 2013/08/M/ST9/00322

\section{REFERENCES}

[1] H. Falcke,W. D. Apel, A. F. Badea, et al., "Detection and imaging of atmospheric radio flashes from cosmic ray air showers", Nature, vol. $\mathbf{4 3 5}$, pp. 313-316, May 2005.

[2] D. Ardouin, A. Bell'etoile, D. Charrier, et al., "Radioelectric field features of extensive air showers observed with CODALEMA" Astropart. Phys. vol. 26, pp. 341-350, Dec. 2006.

[3] J. Abraham et al., [Pierre Auger Collaboration], "Properties and Performance of the Prototype Instrument for the Pierre Auger Observatory", Nucl. Instr. Meth., ser. A, vol. 523, pp. 50-95, May 2004. 


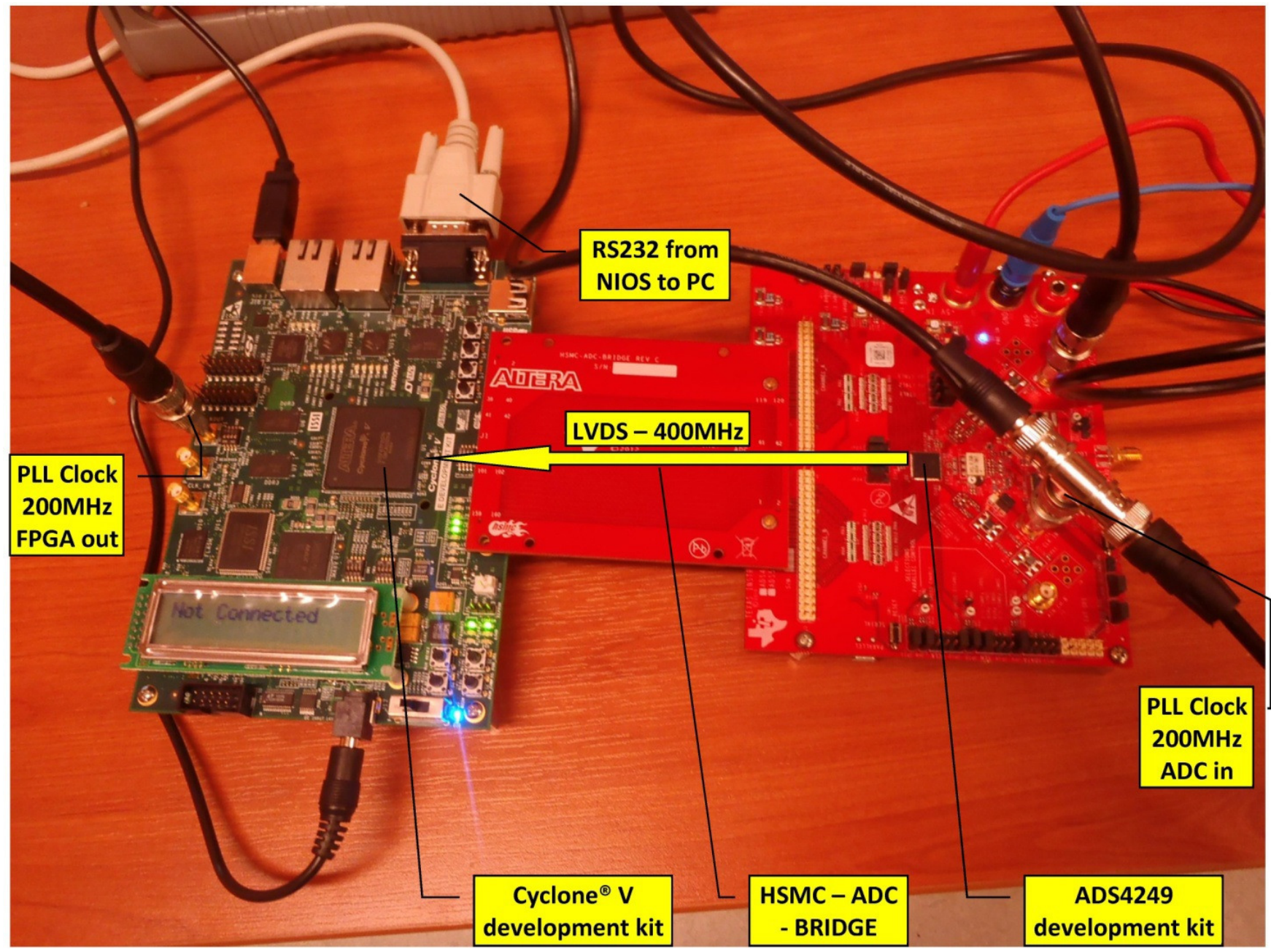

Fig. 22. Connections between developement kit with Cyclone V used for laboratory tests and Texas Instr. ADS4249EVM Evaluation Module. Data were transfered using LVDS technicque by Altera HSMC-ADC-BRIDGE.

[4] S. Fliescher for the Pierre Auger Collaboration, "Radio detection of cosmic ray induced air showers at the Pierre Auger Observatory", Nucl. Instr. Meth., ser. A, vol. 662, pp. 124-129, Jan. 2012.

[5] D. J. Fegan, "Detection of elusive radio and optical emission from cosmicray showers in the 1960s", Nucl. Instr. Meth., ser. A, vol. 662, pp. 2-11, Jan. 2012.

[6] G. A. Askaryan, Journal of Exp. and Theoretical Phys., vol. 14, pp. 441 1962.

[7] G. A. Askaryan, "Coherent Radio Emission from Cosmic Showers in Air and in Dense Media", Journal of Exp. and Theoretical Phys., vol. 21, pp. 658-659, Jan. 1965.

[8] J. Makhoul, "Linear prediction: A tutorial review" Proc. of the IEEE, vol. 63, no. 4, pp. 561-580, Apr. 1975.

[9] Z. Szadkowski, E.D. Fraenkel, A. M. van den Berg, "FPGA/NIOS Implementation of an Adaptive FIR Filter Using Linear Prediction to Reduce Narrow-Band RFI for Radio Detection of Cosmic Rays", IEEE Trans. on Nucl. Science, vol. 60, pp. 3483-3490, Oct. 2013.

[10] Z. Szadkowski, E.D. Fraenkel, D. Głas, R. Legumina, "An optimization of the FPGA/NIOS adaptive FIR filter using linear prediction to reduce narrow band RFI for the next generation ground-based ultra-high energy cosmic-ray experiment", Nucl. Instr. Meth., ser. A, vol. 732, pp. 535-539, June 2013

[11] Z. Szadkowski, Ad M. van den Berg, E.D. Fraenkel, D. Głas, J. Kelley, C. Timmermans, T. Wijnen, "Analysis of the efficiency of the filters suppressing the RFI being developed for the extension of AERA", 33nd International Cosmic Ray Conference - July 2013 - Rio de Janeiro, Brazil.

[12] Z. Szadkowski, D. Głas, C. Timmermans, T. Wijnen for the Pierre Auger Collaboration, "First results from the FPGA/NIOS Adaptive FIR Filter Using Linear Prediction Implemented in the AERA Radio Stations to Reduce Narrow Band RFI for Radio Detection of Cosmic Rays", IEEE Real Time Conference - May 2014 - Nara, Japan.

[13] Z. Szadkowski, D. Głas for the Pierre Auger Collaboration, "Adaptive Linear Predictor FIR Filter based on the Cyclone V FPGA with HPS to Reduce Narrow Band RFI in AERA Radio Detection of Cosmic Rays", Advancements in Nuclear Instrumentations Measuerment Methods and their Applications - April 2015 - Lisbon, Portugal. 